\title{
Sudden Unexplained Death in Childhood
}

National Cancer Institute

\section{Source}

National Cancer Institute. Sudden Unexplained Death in Childhood. NCI Thesaurus. Code C111854.

Unexpected death of a child over one year of age, which remains unexplained when all known and possible causes of death have been ruled out. 